\title{
Article \\ miR-27a Regulates Sheep Adipocyte Differentiation by Targeting CPT1B Gene
}

\author{
Bo Li ${ }^{+}{ }^{+}$, Xiaoyu Huang ${ }^{\dagger}$, Chen Yang, Ting Ge, Leiyun Zhao, Xiaoqiang Zhang, Lintao Tian and Enping Zhang * \\ College of Animal Science and Technology, Northwest A\&F University, Xianyang 712100, China; \\ lib1028@163.com (B.L.); hxy_beyond@sina.com (X.H.); aayangchenaa@163.com (C.Y.); \\ geting@nwafu.edu.cn (T.G.); zly18893818438@163.com (L.Z.); zhangxiaoqiang1028@163.com (X.Z.); \\ tlt2021296@126.com (L.T.) \\ * Correspondence: zhangenping@nwafu.edu.cn \\ † These authors contributed equally to this work.
}

Citation: Li, B.; Huang, X.; Yang, C.; Ge, T.; Zhao, L.; Zhang, X.; Tian, L.; Zhang, E. miR-27a Regulates Sheep Adipocyte Differentiation by Targeting CPT1B Gene. Animals 2022, 12, 28. https://doi.org/10.3390/ ani12010028

Received: 2 November 2021 Accepted: 20 December 2021 Published: 23 December 2021

Publisher's Note: MDPI stays neutral with regard to jurisdictional claims in published maps and institutional affiliations.

Copyright: (C) 2021 by the authors. Licensee MDPI, Basel, Switzerland. This article is an open access article distributed under the terms and conditions of the Creative Commons Attribution (CC BY) license (https:// creativecommons.org/licenses/by/ $4.0 /)$.
Simple Summary: The content of intramuscular fat (IMF) is the main determinant of the nutritional and economic value of sheep meat. Therefore, lipid synthesis in sheep longissimus lumborum (LL) has become an important research focus. MicroRNA-27a (miR-27a) has been shown to play a crucial role in the proliferation and differentiation of adipocyte progenitor cells. In this study, we revealed that miR-27a significantly inhibited the formation of lipid droplets by targeting CPT1B to inhibit genes involved in lipid synthesis including PPAR $\gamma$, SCD, LPL, and FABP4. Here, we constructed a miR-27a-CPT1B regulatory network map, which revealed the interaction between miR-27a and CPT1B in lipid synthesis in ovine preadipocytes.

Abstract: MiRNAs are vital regulators and play a major role in cell differentiation, biological development, and disease occurrence. In recent years, many studies have found that miRNAs are involved in the proliferation and differentiation of adipocytes. The objective of this study was to evaluate the effect of miR-27a and its target gene CPT1B on ovine preadipocytes differentiation in Small-tailed Han sheep (Ovis aries). Down-regulation of miR-27a significantly promoted the production of lipid droplets, while overexpression of miR-27a led to a reduction in lipid droplet production. In addition, inhibition of miR-27a led to a significant increase in the expression of genes involved in lipid synthesis, including PPAR $\gamma$, SCD, LPL, and FABP4. Target Scan software predicted that CPT1B is a new potential target gene of miR-27a. Further experiments revealed that $C P T 1 B$ gene expression and protein levels were negatively correlated with miR-27a expression. Overexpression of miR-27a led to a significant decrease in $C P T 1 B$ mRNA levels and inhibited the accumulation of lipid droplets and vice versa. Moreover, overexpression of $C P T 1 B$ promoted the synthesis of lipid droplets in ovine preadipocytes. Furthermore, luciferase reporter assays confirmed CPT1B to be a miR-27a direct target gene. This study confirmed that miR-27a increases the expression of genes related to lipid synthesis in ovine preadipocytes by targeting $C P T 1 B$, thereby promoting the synthesis of lipid droplets. The results of this study can be used to be exploited in devising novel approaches for improving the IMF content of sheep.

Keywords: miR-27a; CPT1B; ovine preadipocytes; adipogenesis

\section{Introduction}

The content of intramuscular fat (IMF) content is an index to measure meat quality [1], which directly affects the tenderness, juiciness, and flavor of mutton [2]. Therefore, understanding the mechanism of IMF synthesis in sheep meat has become a research hotspot. The formation of IMF in sheep is regulated by many factors, such as heredity [3], age [4], environment [5], and nutrition [6]. However, the molecular mechanism underlying IMF formation is still poorly understood. 
MicroRNAs (miRNAs) are one of the most important regulatory factors [7] by repressing mRNA translation or by binding to target mRNAs [8]. In addition, it has been found that miRNAs play a key role in fat synthesis. For instance, miR-340-5p regulates lipid formation by targeting ATF7 [9]. Moreover, miR-32-5p has been shown to promote adipocyte differentiation via inhibition of KLF3 [10]. miR-124-3p targets $C / E B P \alpha$ and influences fat differentiation in sheep muscle tissue [11]. miR-330-5p negatively regulates adipocyte differentiation by targeting the BCAT2 gene [12].

As a member of the miRNA-27 family, miR-27a regulates the differentiation of ovine preadipocytes and triglycerides synthesis by regulating the expression of the target gene $R X R$ [13]. MiR-27a is a regulatory factor of peroxisome proliferator-activated receptor $\mathrm{g}$ $(P P A R) \gamma$ [14]. It has been hypothesized that CPT1B may be the target gene of miR-27a. Interestingly, studies have shown that $C P T 1 B$ affects lipid metabolism in Chinese Simmental cattle [15] and that the expression of $C P T 1 B$ is associated with preventing fatty liver disease [16].

Therefore, in this study, the relationship between miR-27a and CPT1B was studied. In addition, the impact of the miR-27a-CPT1B interaction on the differentiation of ovine preadipocytes was evaluated. This study provides a basis for exploring the role of miR-27a in the differentiation of ovine preadipocytes.

\section{Materials and Methods}

\subsection{Ovine Preadipocytes Isolation and Culture}

Under sterile conditions, small vessels and other connective tissues of LL tissues were removed from one-month-old Small-tailed Han sheep (Yangling, Shaanxi, China). The Small-tailed Han sheep is ewe, weighing $10.8 \mathrm{~kg}$. Muscle tissue was minced, digested, filtered, and centrifuged at $1200 \mathrm{rpm}$ for $10 \mathrm{~min}$. Harvested ovine preadipocytes were cultured as previously described [17].

Preadipocyte differentiation was initiated using an induction medium (Cyagen Biosciences, Santa Clara, CA, USA). The induction medium was composed of medium A (fetal bovine serum (FBS), penicillin/streptomycin, insulin, glutamine, 3-isobutyl 1methylxanthine, rosiglitazone, and dexamethasone) and medium B (FBS, penicillin/ streptomycin, and insulin), which were alternately used every two days until day 8 for cell culture.

\subsection{Cloning of $C P T 1 B$}

The coding sequence of CPT1B of Small-tailed Han sheep was amplified by PCR using KOD-Plus-Neo (Toyobo, Osaka, Japan). TRIzol reagent (Agbio, Changsha, China) was used for RNA isolation from LL of Small-tailed Han sheep, as per the manufacturer's instructions. To synthesize cDNA, a high-capacity cDNA reverse transcription kit (Takara, Dalian, China) was used. cDNA was used as a template. CPT1B fragment was amplified using the following primers: forward primer 5'- ATGACAACAGTGGGTTCCTCCTG $3^{\prime}$ and reverse primer 5'- TTAGCCATCAGCCTTAGAAACTTG $-3^{\prime}$. PCR products were cloned into the pcDNA3.1 vector and sequenced at ZKYTONG Biological Technology (Xi'an, China). The cloned fragment was named $p c D N A 3.1-C P T 1 B$.

\subsection{Cell Transfection}

Ovine preadipocytes grew to $80 \%$ confluence. MiR-27a simulant, negative control (NC) simulant (miR-NC), miR-27a inhibitor, NC inhibitor (Shanghai Gene Pharmaceuticals, China), and $p c D N A 3.1-C P T 1 B$ were transfected into cells with Lipofectamine ${ }^{\circledR} 3000$ reagent (Invitrogen, Waltham, MA, USA) according to the manufacturer's instructions. After $6 \mathrm{~h}$ of transfection, cell culture was continued in a fresh complete medium, which was replaced by DMEM medium containing 10\% FBS and 1\% penicillin/streptomycin. 


\subsection{Determination of Gene Expression}

$\beta$-actin gene was used as an internal control to determine mRNA expression, however, the U6 gene was used as an internal control for determining miRNA expression [18]. Primers used in real-time PCR were designed and synthesized by ZKYTONG (China) (Table 1). RT-qPCR was carried out in triplicates using the $2 \times$ ChamQ SYBR qPCR Master system (Vazyme, Nanjing, China). The reaction was carried out in a LightCycler ${ }^{\circledR}$ real-time fluorescence-based quantitative PCR system (Roche Life Science, Basel, Switzerland). The $2^{-\Delta \Delta \mathrm{Ct}}$ method was applied to calculate relative gene expression [19].

Table 1. The sequence of primers used in the study for mRNA and miRNA quantitative real-time PCR.

\begin{tabular}{|c|c|c|}
\hline Gene Target & Primer Sequence $\left(5^{\prime}-3^{\prime}\right)$ & Gene ID \\
\hline \multirow{2}{*}{$\beta$-actin(internal reference of mRNA) } & F: ACCGTGAGAAGATGACCCAGA & \multirow{2}{*}{443052} \\
\hline & R: AGAGGCGTACAGGGACAGCA & \\
\hline \multirow{2}{*}{$P P A R \gamma$} & F: TGGATGACCACTCCCATGCC & \multirow{2}{*}{443513} \\
\hline & R: TTGGGAACGGAATGTCCTC & \\
\hline \multirow{2}{*}{$F A B P 4$} & F: GGATGATAAGCTGGTGCTGG & \multirow{2}{*}{100137067} \\
\hline & R: CTCTGGTAGCAGTGACACCG & \\
\hline \multirow{2}{*}{$S C D$} & F: TTCATCCTGCCCACACTCG & \multirow{2}{*}{443185} \\
\hline & R: TAGTTGTGGAAGCCCTCACC & \\
\hline \multirow{2}{*}{$L P L$} & F: CCCAGCAGCATTATCCAGTGT & \multirow{2}{*}{443408} \\
\hline & R: ATTCATCCGCCATCCAGTTC & \\
\hline \multirow{2}{*}{ miR-27a } & F: TCGGCAGGTTCACAGTGGCTA & \multirow{2}{*}{102465824} \\
\hline & R: CTCAACTGGTGTCGTGGAGTC & \\
\hline \multirow{2}{*}{ U6 (internal reference of mRNA) } & F: CAAGGGCCACATAGATCCG & \multirow{2}{*}{101121962} \\
\hline & R: AACGCTTCACGAATTTGCGT & \\
\hline \multirow{2}{*}{ CPT1B } & F: AGATCCGTATGTTCGACCCAA & \multirow{2}{*}{443193} \\
\hline & R: CTGCGATCATGTAGGAAACACC & \\
\hline
\end{tabular}

2.5. Quantitative Assessment of Adipocyte Differentiation Using Lipid (Oil Red O) Staining Assay

Transfected ovine preadipocytes (miR-27a simulant, negative control (NC) simulant (miR-NC), miR-27a inhibitor, NC inhibitor) were induced to differentiate 8 days later. Cells were washed with PBS three times, fixed in 4\% formaldehyde formalin for $30 \mathrm{~min}$, and submitted to staining with Oil Red O (Oro) working solution (60\% saturated solution and $40 \% \mathrm{ddH}_{2} \mathrm{O}$ ) [20].

For measuring intracellular lipid content, stained ovine preadipocytes were eluted with pure isopropanol for $1 \mathrm{~h}$, and sample absorbance was measured at $510 \mathrm{~nm}$ in an Epoch spectrophotometer (BioTek, Winooski, VT, USA).

\subsection{Luciferase Reporter Assay}

MiR-27a target gene and miRNA binding sites were identified using the Target Scan software (http:/ / www.targetscan.org/vert_72/, accessed on 25 February 2021) [21]. The mature sequence of miR-27a was obtained from the miRBase database. Reporter constructs for luciferase were generated $3^{\prime}$-UTR wild-type (WT) and mutation type (MUT) of CPT1B sequences synthesized by ZKYTONG (China) and cloned into the psiCHECK-2 vector (Promega, Madison, WI, USA) at the BamHI sites. Ovine preadipocytes were grown in 12 -well plates transfected at 70\% confluence using Lipofectamine 3000 reagent (Invitrogen). CPT1B-3'UTR WT (0.16 $\mu \mathrm{g})+$ miR-27a-NC (5 pmol), CPT1B-3'UTR WT (0.16 $\mu \mathrm{g})+$ miR-27a (5 pmol), CPT1B-3'UTR MUT $(0.16 \mu \mathrm{g})+$ miR-27a-NC (5 pmol) and CPT1B-3'UTR MUT $(0.16 \mu \mathrm{g})+\mathrm{miR}-27 \mathrm{a}(5 \mathrm{pmol})$ were transfected into wells containing ovine preadipocytes. 
Luciferase report assay system (Beyotime, Shanghai, China) was used to evaluate luciferase activity $48 \mathrm{~h}$ after transfection.

\subsection{Western Blot Assay}

Ovine preadipocytes were collected using 0.25\% trypsin (Solarbio, Beijing, China) and lysed in RIPA buffer (Solarbio) containing 1\% PMSF (Pierce, Rockford, IL, USA). Western blot was carried out as described previously [22]. Primary antibodies against $\beta$-actin (Abcam 8226) (1:2000, Abcam), CPT1B (K004803P) (1:1000, Solarbio), and HRP goat anti-Rabbit IgG secondary antibody (ab97051) (1:2000, Abcam) were used. Chemiluminescent ECL Western blot system (Pierce, Rockford, IL, USA) was used for measuring signal detection.

\subsection{Data Analysis}

Data analysis was performed in GraphPad v.8 (GraphPad Software Inc., San Diego, CA, USA). Data are presented as mean \pm SD. Each experiment was performed in triplicate. Student's t-test was used for pairwise comparisons. Statistical significance was considered at values of $p<0.05, p<0.01$, and $p<0.001$.

\section{Results}

\subsection{Expression of miR-27a and CPT1B under Ovine Preadipocytes Differentiation}

Ovine preadipocytes were successfully isolated from the LL of Small-tailed Han sheep and submitted to induced differentiation. Temporal patterns of miR-27a and CPT1B expression were assessed during ovine preadipocytes adipogenesis. During ovine preadipocytes differentiation, expression of miR-27a gradually decreased (Figure 1A), whereas expression of $C P T 1 B$ gradually increased (Figure $1 B$ ). These results suggest that miR-27a displayed a contrary expression pattern with $C P T 1 B$ during ovine preadipocytes differentiation.

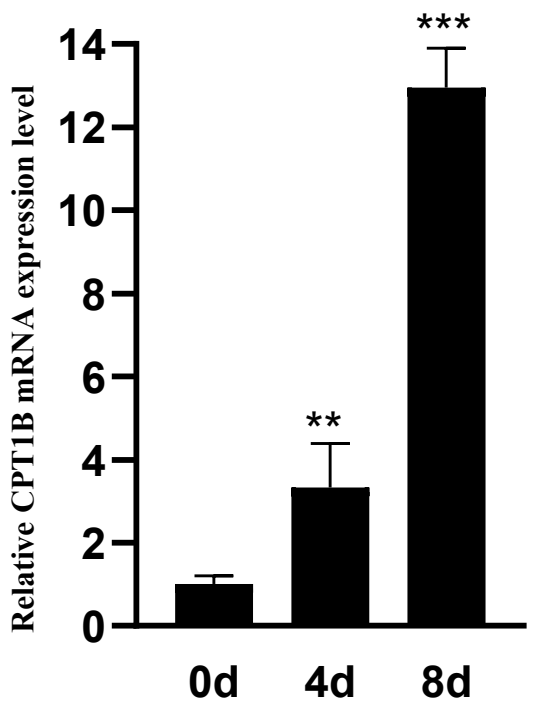

(A)

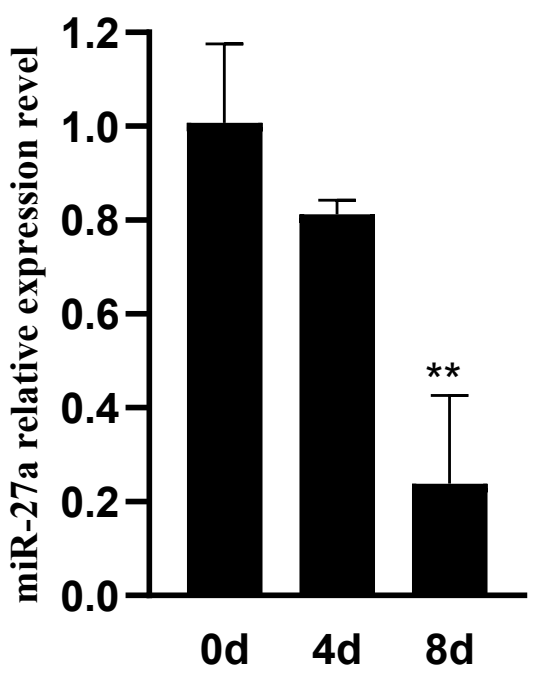

(B)

Figure 1. Temporal expression pattern of miR-27a and CPT1B under ovine preadipocytes differentiation. Ovine preadipocytes were submitted to induced differentiation for eight days. Cells were collected on days 0,4 , and 8 , and expression of CPT1B (A) and miR-27a (B) was determined by RT-PCR. ${ }^{* *} p<0.01,{ }^{* * *} p<0.001$.

\subsection{MiR-27a Inhibits Differentiation of Ovine Preadipocytes}

The efficiency of miR-27a mimic and inhibitor transfection was confirmed by RT-PCR. miR-27a simulant significantly increased expression of miR-27a $(p<0.001)$ (Figure 2A), while miR-27a inhibitor significantly inhibited expression of miR-27a $(p<0.05)$ (Figure 3A). 
(A)

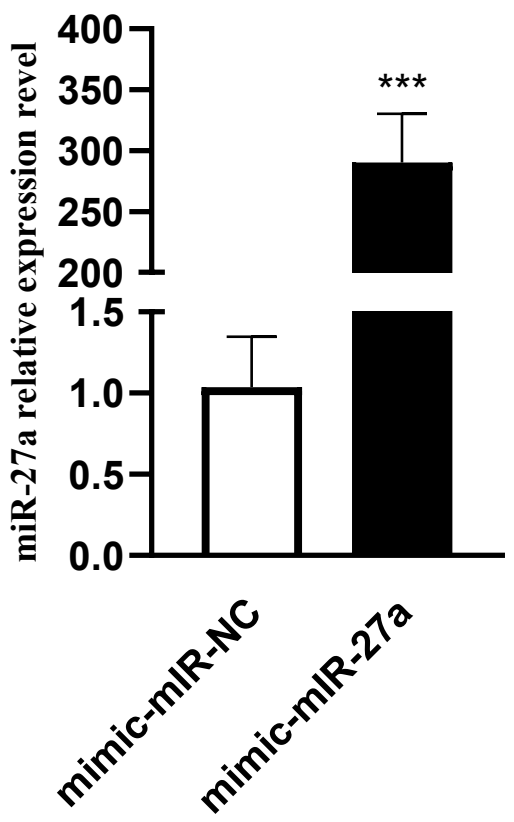

(B)

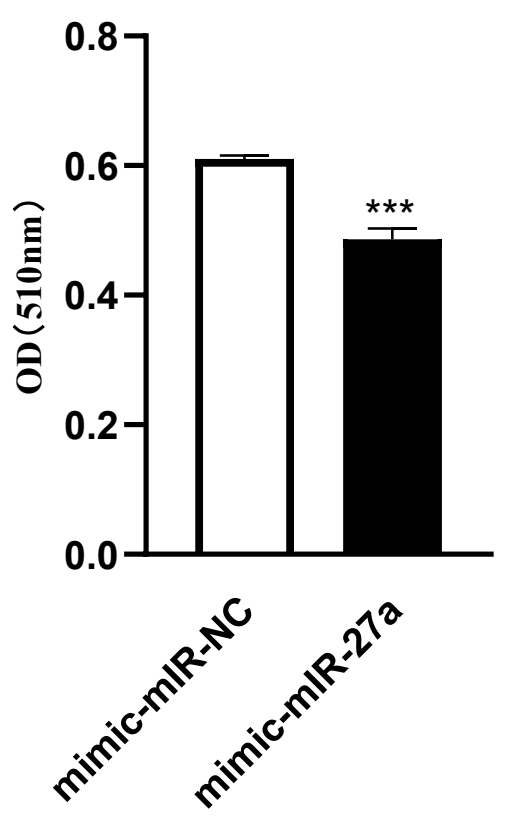

(C)

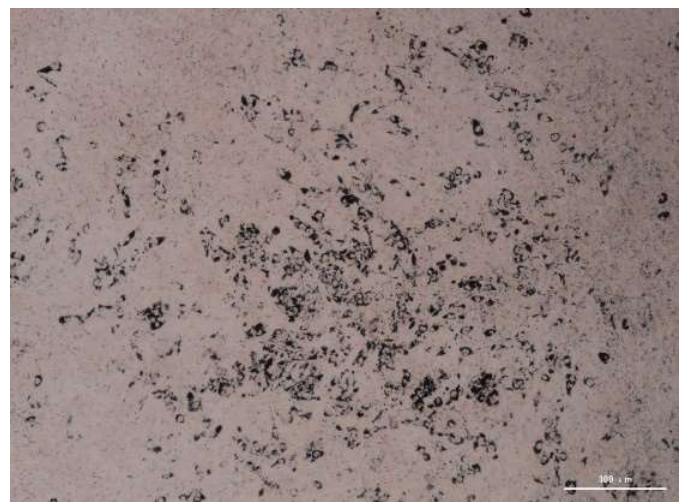

miR-mimic-NC

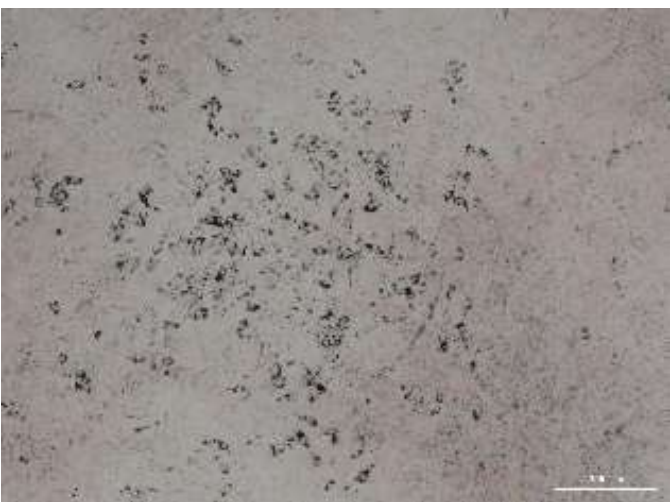

miR-mimic-27a

Figure 2. Effect of the miR-27a mimic on ovine preadipocytes. (A) miR-27a expression following miR-27a mimic treatment; (B) cellular lipid content as determined by spectrophotometry; (C) change in lipid droplet accumulation in ovine preadipocytes following miR-27a mimic treatment. *** $p<0.001$.

To determine the role of miR-27a in lipid metabolism, the absorbance of the sample at 510nm was determined to measure the content of intracellular lipids, and lipid droplet accumulation following overexpression or silencing of miR-27a was determined. miR27a mimic significantly inhibited the accumulation of lipid droplets compared with the control group $(p<0.001)$ (Figure 2B,C). In contrast, the number of lipid droplets increased significantly when miR-27a was inhibited $(p<0.05)$ (Figure 3B,C). Overall, these findings indicate that miR-27a inhibits the synthesis of lipid droplets in ovine preadipocytes. 
(A)

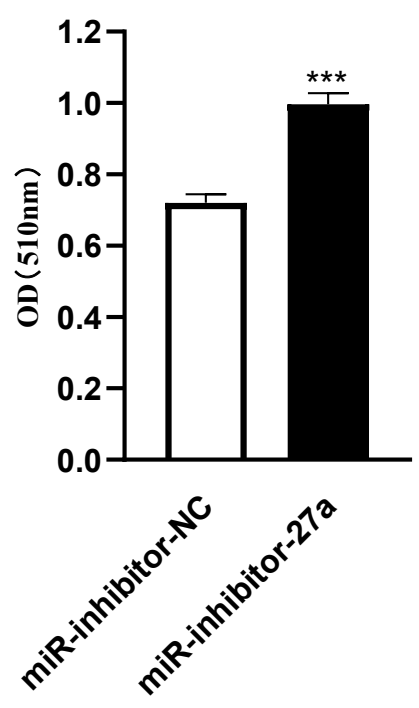

(B)

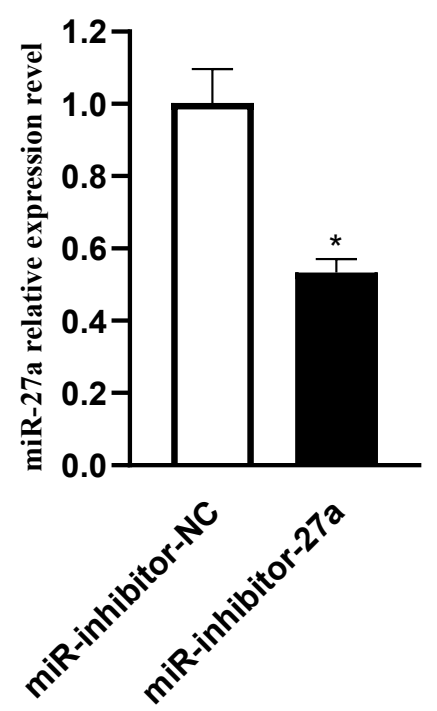

(C)

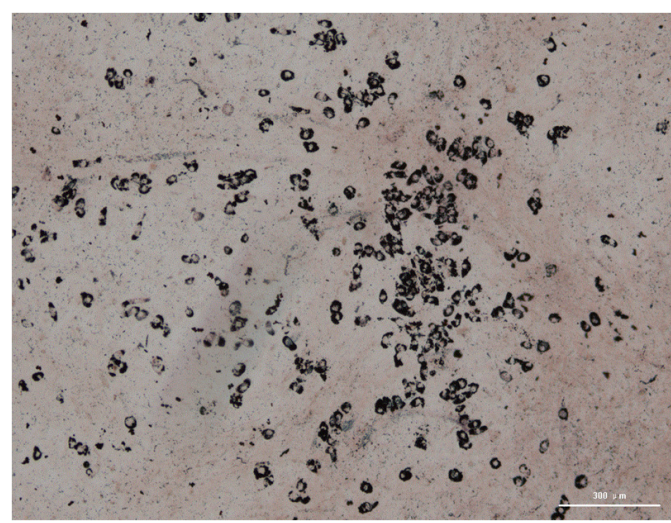

miR-inhibitor-NC

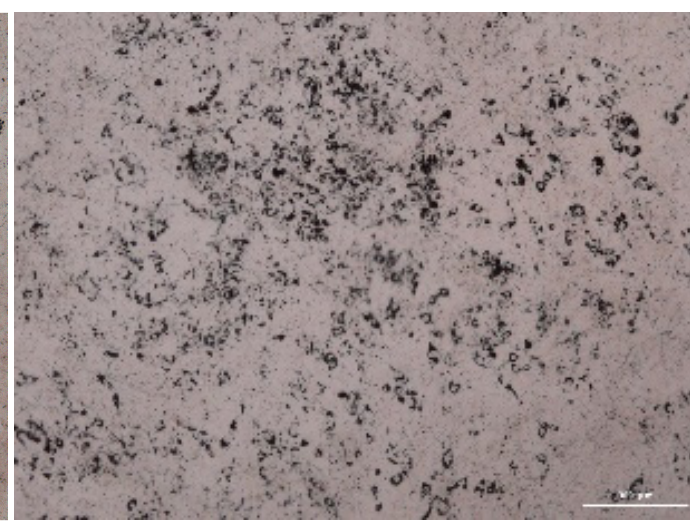

miR-27a-inhibitor

Figure 3. Effect of miR-27a inhibitor on ovine preadipocytes. (A) miR-27a expression following miR-27a inhibitor treatment; (B) cellular lipid content as determined by spectrophotometry; (C) change in lipid droplet accumulation in ovine preadipocytes after treatment with miR-27a inhibitor. ${ }^{*} p<0.05$; ${ }^{* *} p<0.001$.

\subsection{MiR-27a Regulates Expression of Genes Related to Lipid Metabolism in Ovine Preadipocytes}

MiR-27a overexpression significantly reduced expression of genes associated with lipid synthesis, such as PPAR $\gamma(p<0.001), S C D(p<0.001), L P L(p<0.001)$, and FABP4 $(p<0.001)$ (Figure 4A). Conversely, transfection of miR-27a inhibitor significantly promoted expression of PPAR $\gamma(p<0.001), S C D(p<0.01), L P L(p<0.05)$, and FABP4 $(p<0.001)$ (Figure 4B). Collectively, it can be inferred that miR-27a had a negative effect on the expression of genes related to fat synthesis in ovine preadipocytes. 
(A)

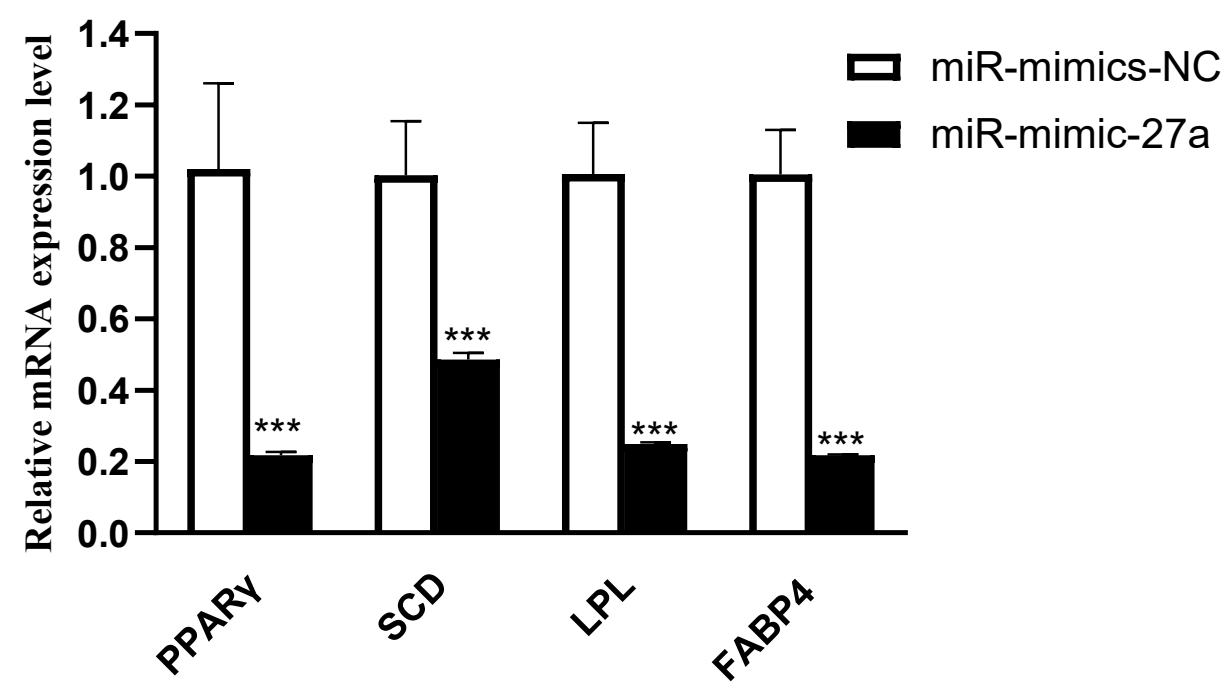

(B)

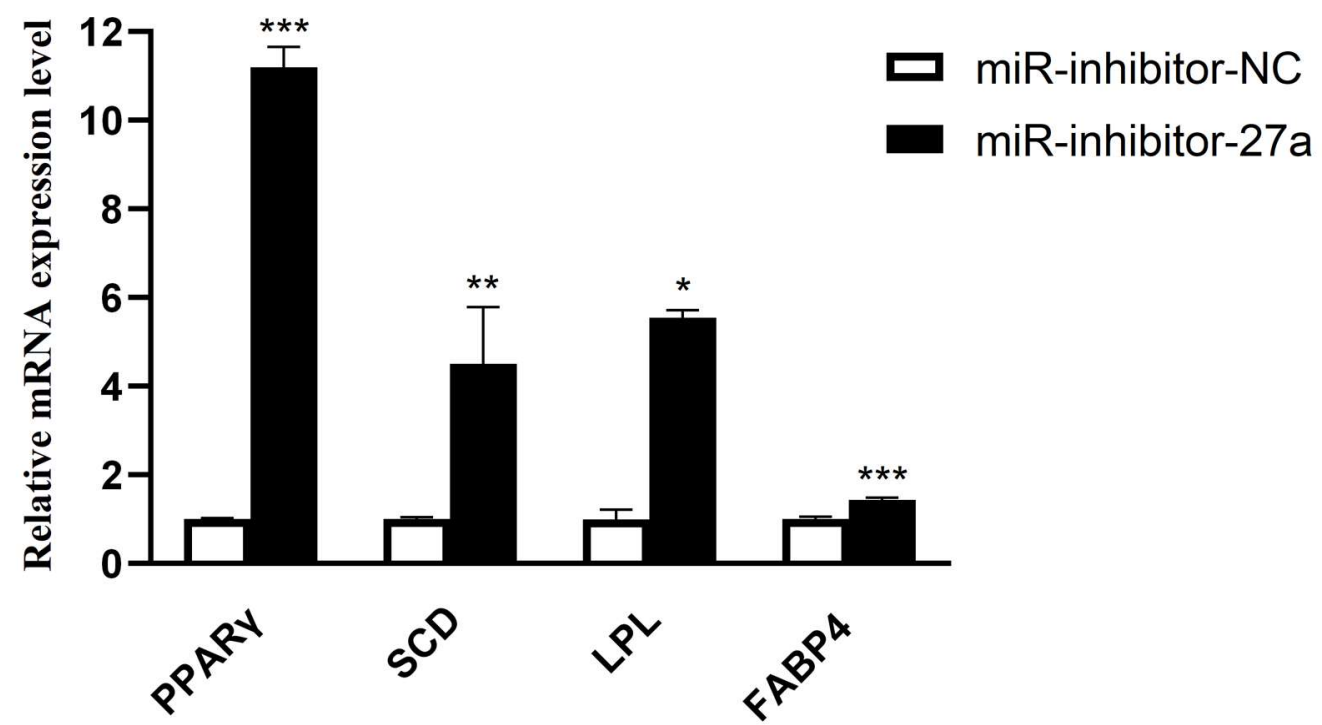

Figure 4. Effect of miR-27a on expression of genes related to lipid metabolism. (A) Ovine preadipocytes transfected with miR-27a mimic; $($ B) ovine preadipocytes transfected with miR-27a inhibitor. ${ }^{* *} p<0.001$.

\subsection{CPT1B Is a Target Gene for miR-27a}

As shown in Figure 5A, treatment with miR-27a mimic led to downregulation of $C P T 1 B$ expression. In contrast, treatment with miR-27a inhibitor remarkably enhanced CPT1B expression $(p<0.01)$ (Figure $5 B)$. Simultaneously, Western blot assay revealed that levels of $C P T 1 B$ protein in miR-27a mimic and inhibitor transfection groups were comparable to those of mRNA expression. Therefore, it can be stated that miR-27a reduced $C P T 1 B$ mRNA levels and protein levels in ovine preadipocytes. 
(A)
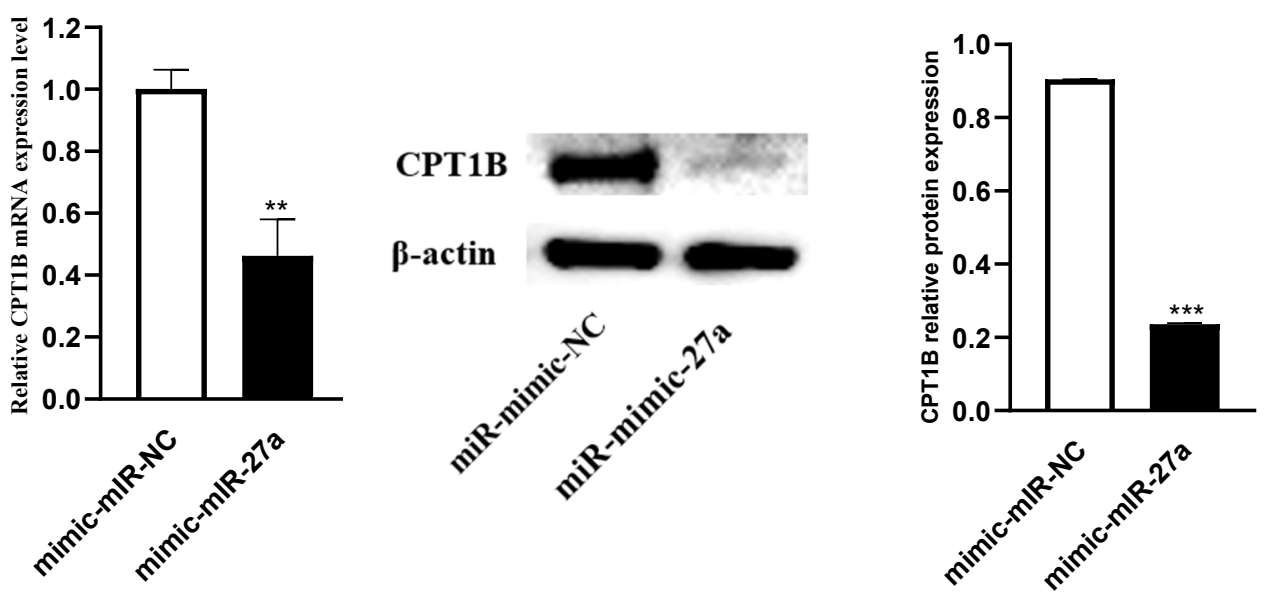

(B)
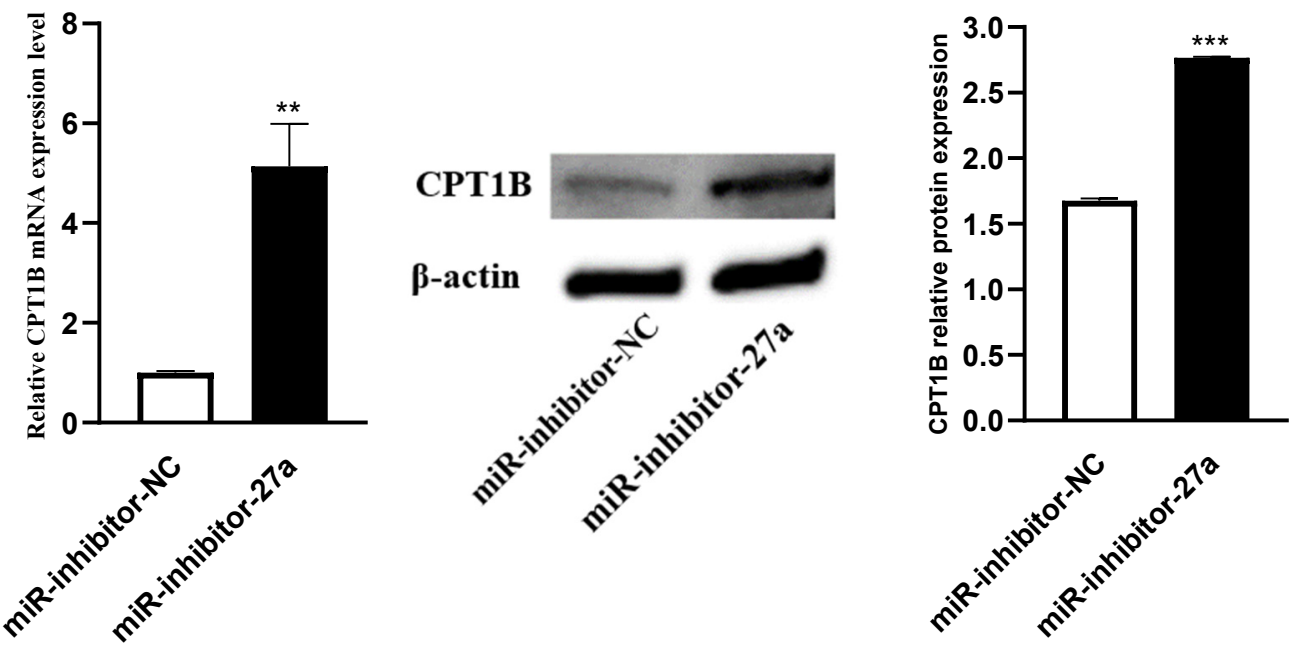

Figure 5. Impact of miR-27a on the $C P T 1 B$ mRNA levels and protein expression. (A) Effects of transfection of miR-mimic-NC and miR-mimic-27a on CPT1B gene expression and protein in adipocytes. (B) Effects of transfection of miR-inhibitor-NC and miR-inhibitor-27a on CPT1B gene expression and protein in adipocytes. ${ }^{* *} p<0.01,{ }^{* * *} p<0.001$.

To further investigate the mechanism underlying miR-27a inhibition of fat deposition in sheep muscle tissue, we aimed to investigate whether miR-27a targets the 3'UTR region of $C P T 1 B$, this region had the binding site for the seed sequence of miR-27a (Figure 6A). Therefore, the luciferase reporter system was used to confirm whether $C P T 1 B$ is a target gene for miR-27a. Co-transfection of miR-27a mimics and CPT1B-WT-miR-27a vector directly repressed luciferase activity (Figure $6 \mathrm{~B} ; p<0.01$ ), which suggests that $C P T 1 B$ is a direct target gene of miR-27a. Overall, these findings revealed that miR-27a inhibited differentiation and promoted proliferation in ovine preadipocytes by negatively regulating $C P T 1 B$. 
(A)

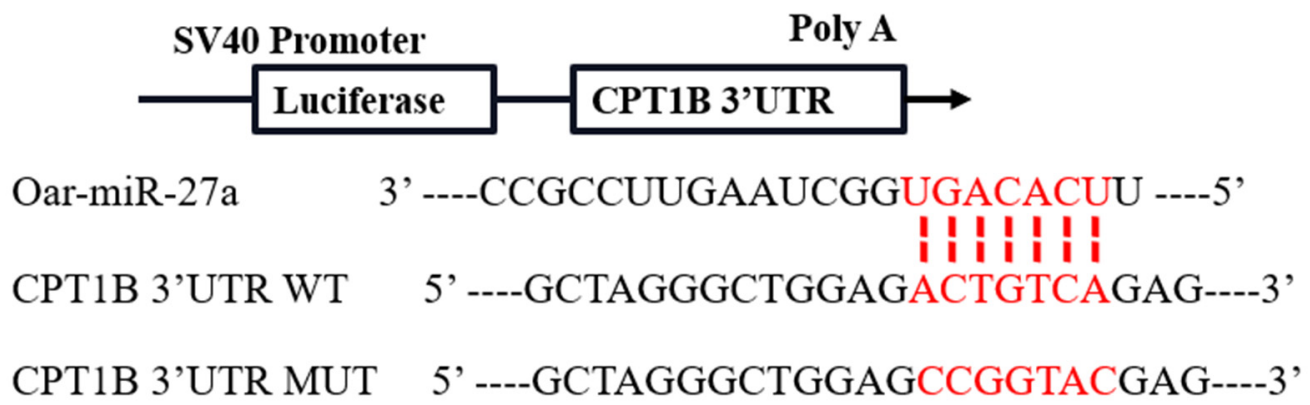

(B)

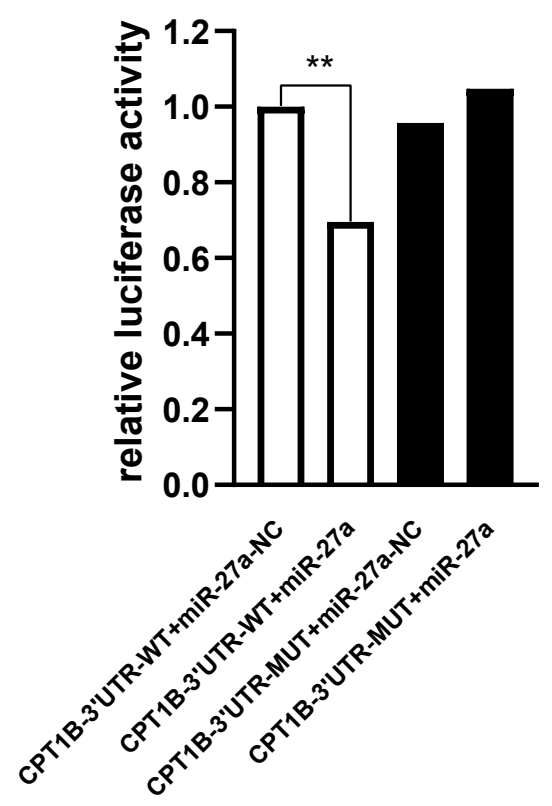

Figure 6. (A) Sequence alignment of miR-27a and the $3^{\prime}$-untranslated region (UTR) of $C P T 1 B$ as determined by Target Scan software and miRDB target prediction database; (B) changes in luciferase activity after ovine preadipocytes cells were co-transfected with miR-27a mimic and a luciferase reporter with a fragment of the CPT1B $3^{\prime}$-UTR harboring either the miR-27a binding site (CPT1B-3'UTR-WT) or a mutant $\left(C P T 1 B-3^{\prime}\right.$ UTR-MUT). ${ }^{* *} p<0.01$. SV40: Simian virus 40; poly A: polyadenylic acid.

\subsection{Effect of $C P T 1 B$ Overexpression on Ovine Preadipocytes Differentiation}

To reveal the role of $C P T 1 B$ in lipid metabolism of ovine preadipocytes, a CPT1B overexpression vector was constructed and transfected into ovine preadipocytes. The optimal transfection concentration was $2.5 \mu \mathrm{g}$ to yield the highest expression efficiency. Compared with the control group, levels of $C P T 1 B$ mRNA and protein expression in transfected ovine preadipocytes increased significantly $(p<0.05)$ (Figure 7A,B). After 8 days of induction, $C P T 1 B$ overexpression promoted lipid accumulation in ovine preadipocytes (Figure 7C). Collectively, these findings suggest that overexpression of $C P T 1 B$ in ovine preadipocytes promotes adipogenesis. 
(A)

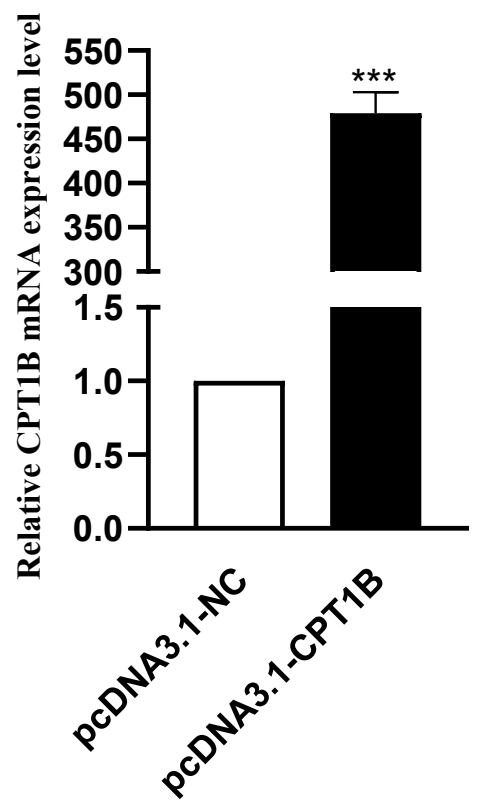

(B)
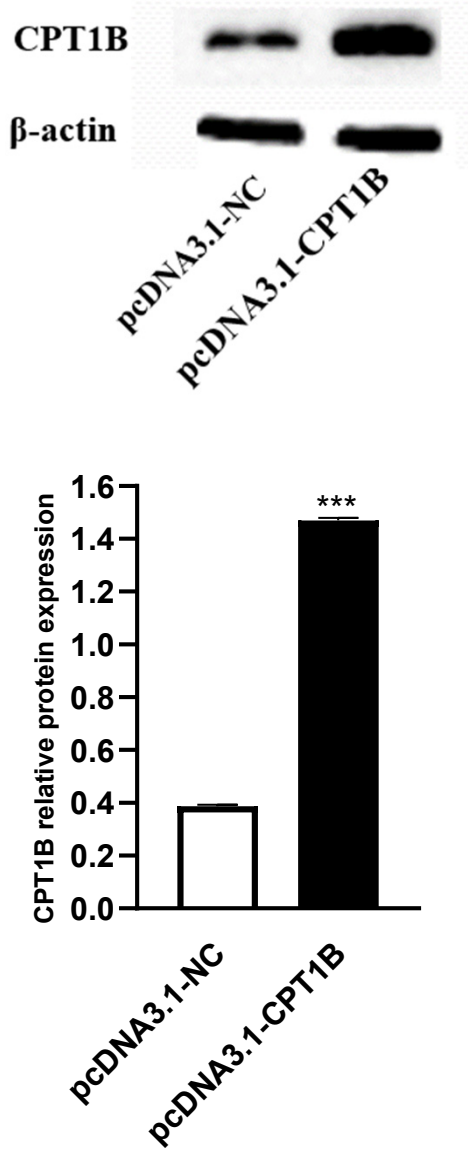

(C)

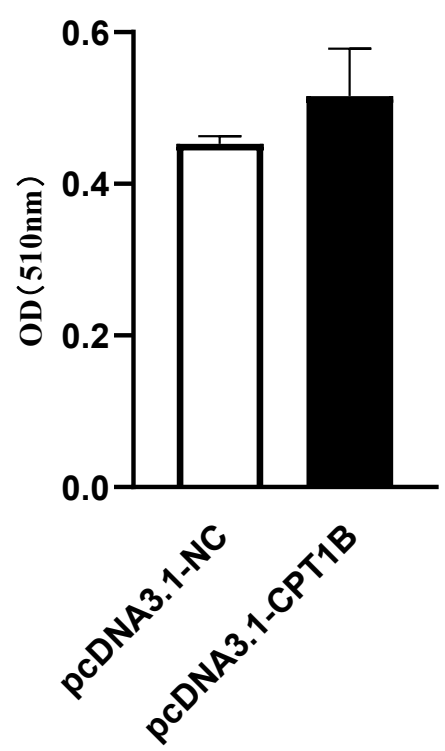

Figure 7. Effect of $C P T 1 B$ on ovine preadipocytes differentiation. (A,B) Comparative expression of $C P T 1 B$ mRNA and protein levels in ovine preadipocytes transfected with overexpression CPT1B vector and $p c D N A 3.1-N C$ for $48 \mathrm{~h}$; (C) cellular lipid content as determined by spectrophotometry. $* * *<0.001$. 


\section{Discussion}

$C P T 1 B$ is a major isotype of the $C P T$ family which comprises other two genes, namely CPT1A and CPT2 [23]. CPT1B gene plays a vital role in regulating fat decomposition and energy supply. Studies have shown that $C P T 1 B$ is of great significance to the development of brown adipose tissue [24]. Moreover, in bovine fetal fibroblasts, changes in triglyceride content were related to the expression level of $C P T 1 B$ [25]. It has also been demonstrated that downregulation of $C P T 1 B$ leads to impaired fatty acid oxidation [26]. Furthermore, decreased $C P T 1 B$ protein levels limit the oxidation of cardiac fatty acids [27]. In the present study, overexpression of $C P T 1 B$ led to a significant increase in lipid accumulation in ovine preadipocytes. Interestingly, previous studies have reported that miR-27a inhibits ovine preadipocytes differentiation by regulating the expression of the target gene $R X R$ alpha [13]. CPT1B is downstream RXR alpha in the PPAR $\gamma$ signaling pathway. Therefore, it can be speculated that miR-27a may regulate lipid deposition in ovine preadipocytes via the PPAR $\gamma$ signaling pathway. However, the specific mechanism of regulation needs further elucidation. Based on the above-described results, it can be inferred that miR27a regulates lipid deposition in ovine preadipocytes by targeting CPT1B. Animal fat metabolism is regulated by many factors [28-30]. Abnormal fat metabolism leads to increased incidence of a variety of metabolic diseases [31,32]. Therefore, understanding the mechanism underlying the regulation of lipid metabolism has always been the focus of several studies. In recent years, research on miRNA has confirmed that a large number of miRNAs are involved in the regulation of many biological processes related to adipose tissue development [33-35]. In previous studies, it has been suggested that CPT1B can be a candidate gene for fat deposition in sheep adipose tissue. In addition, it has been hypothesized previously that $C P T 1 B$ may be a potential target gene for miR-27a. In the present study, ovine preadipocytes were employed as a model to explore the regulatory mechanism between miR-27a and CPT1B in adipose tissue differentiation, which provided a theoretical basis for further understanding the effect of miRNA on lipid formation in ovine preadipocytes.

The regulatory effect of miR-27a on many types of cells has been previously reported. For instance, silencing miR-27a promoted autophagy and apoptosis of melanoma cells [36], whereas overexpression of miR-27a targeted GSK-3 $\beta$ and promoted breast epithelial cell proliferation and invasion [37]. Moreover, miR-27a-3p reduced ATF3 mRNA and protein levels in a cellular vascular calcification model. [38]. Additionally, miR-27a is closely related to lipid synthesis [39]. MiR-27a affects lipid deposition in IMF and subcutaneous adipose tissue of sheep by participating in adipocyte differentiation and triglyceride synthesis [40,41]. miR-27a and miR-27b target expression of PPAR $\gamma$ in 3T3-L1 cells, thereby controlling the production of lipid droplets [42]. MiR27a can also inhibit NRF2 expression and contribute to lipid accumulation in hepatocytes [43]. Collectively, the results discussed herein contribute to show that miR-27a negatively regulates lipid production in ovine preadipocytes.

In addition, in the present study, the expression level of miR-27a decreased with differentiation and maturation of ovine preadipocytes, whereas the expression level of $C P T 1 B$ increased with the differentiation and maturation of ovine preadipocytes. These findings are consistent with previous works which showed that the expression level of the CPT1B gene increased with an increase in the accumulation of lipid droplets in bovine fetal fibroblasts [25]. The expression trend of miR-27a is opposite to the marker gene PPAR $\gamma$ for adipogenic differentiation $[44,45]$, whereas PPAR $\gamma$ expression level is positively correlated with ovine preadipocytes differentiation. Collectively, these results suggest that miR-27a negatively regulates differentiation of and lipid accumulation in ovine preadipocytes.

Herein, miR-27a was found to inhibit significantly lipid droplet accumulation in ovine preadipocytes, which is consistent with previous studies showing that miR-27a negatively regulates lipid production in ovine preadipocytes [13]. Furthermore, miR-27a was shown to decrease expression of PPAR $\gamma, S C D, L P L$, and FABP4 which are genes related to lipid synthesis in ovine preadipocytes. PPAR $\gamma$ is a positive regulator of lipid accumulation which plays a key regulatory role in adipocyte differentiation [46]. FABP4 has a significant 
role in fat deposition and is highly expressed in fat tail [47]. Previous studies showed a possible effect of SCD on the regulation of milk fat percentage in Churra sheep [48]. LPL plays an important role in regulating fat synthesis in goat breast milk [49]. Our results, therefore, suggest that miR-27a may inhibit the synthesis of lipid droplets by affecting key genes involved in lipid regulation.

\section{Conclusions}

In the present study, miR-27a was shown to be involved in fat synthesis and accumulation in ovine preadipocytes. Specifically, miR-27a regulated the formation of lipid droplets in adipocytes by targeting the $C P T 1 B$ gene. These results may provide a theoretical basis for understanding IMF deposition in sheep muscle tissue.

Author Contributions: B.L. conceived the study and designed the experiments, performed the experiments, analyzed the data, wrote the manuscript. X.H. and B.L. contributed equally to this work, conceived the study and analyzed the data. C.Y. helped with animal samples collection and performed the experiments. T.G. revised the paper. L.Z. supplied the materials. X.Z. supplied the materials. L.T. helped with animal samples collection. E.Z. conceived the study, contributed to the final version of the manuscript and supervised the study. All authors have read and agreed to the published version of the manuscript.

Funding: Research supported by the Shaanxi mutton sheep industry technology system construction project (NYKJ-2020-YL-14 and NYKJ-2021-YL (XN) 43) and Breeding of new meat sheep varieties (strains) \& research and Demonstration of efficient breeding technology of hybrid meat sheep (NYKJ2019-YL35).

Institutional Review Board Statement: The study was conducted according to the institutional animal care and committee (IACUC) guidelines and approved by the Animal Care and Use Committee of Northwest A\&F University, Yangling, China (NWAFU-20201212).

Data Availability Statement: The datasets used and/or analyzed during the current study available from the corresponding author on reasonable request.

Acknowledgments: Thanks very much for the venue provided by Gansu Qinghuan mutton sheep Seed Production Co., Ltd. We express our thanks to all members in Y. Chen's laboratory who contributed to this study.

Conflicts of Interest: The authors declare no conflict of interest.

\section{References}

1. Hocquette, J.F.; Gondret, F.; Baéza, E.; Médale, F.; Jurie, C.; Pethick, D.W. Intramuscular fat content in meat-producing animals: Development, genetic and nutritional control, and identification of putative markers. Animal 2010, 4, 303-319. [CrossRef]

2. Frank, D.; Watkins, P.; Ball, A.; Krishnamurthy, R.; Piyasiri, U.; Sewell, J.; Ortuño, J.; Stark, J.; Warner, R. Impact of Brassica and lucerne finishing feeds and intramuscular fat on lamb eating quality and flavor. A cross-cultural study using Chinese and Non-Chinese Australian consumers. J. Agric. Food Chem. 2016, 64, 6856-6868. [CrossRef] [PubMed]

3. Liang, C.; Qiao, L.; Han, Y.; Liu, J.; Zhang, J.; Liu, W. Regulatory Roles of SREBF1 and SREBF2 in lipid metabolism and deposition in two Chinese representative fat-tailed sheep breeds. Animals 2020, 10, 1317. [CrossRef] [PubMed]

4. Watkins, P.J.; Rose, G.; Salvatore, L.; Allen, D.; Tucman, D.; Warner, R.D.; Dunshea, F.R.; Pethick, D.W. Age and nutrition influence the concentrations of three branched chain fatty acids in sheep fat from Australian abattoirs. Meat Sci. 2010, 86, 594-599. [CrossRef]

5. $\quad$ Fan, H.; Zhang, Y.; Zhang, J.; Yao, Q.; Song, Y.; Shen, Q.; Lin, J.; Gao, Y.; Wang, X.; Zhang, L.; et al. Cold-inducible Klf9 regulates thermogenesis of brown and beige fat. Diabetes 2020, 69, 2603-2618. [CrossRef]

6. Khanal, P.; Pandey, D.; Binti Ahmad, S.; Safayi, S.; Kadarmideen, H.N.; Olaf Nielsen, M. Differential impacts of late gestational over-and undernutrition on adipose tissue traits and associated visceral obesity risk upon exposure to a postnatal high-fat diet in adolescent sheep. Physiol. Rep. 2020, 8, e14359. [CrossRef]

7. Lu, T.X.; Rothenberg, M.E. MicroRNA. J. Allergy Clin. Immunol. 2018, 141, 1202-1207. [CrossRef]

8. Mohr, A.M.; Mott, J.L. Overview of microRNA biology. Semin. Liver Dis. 2015, 35, 3-11. [CrossRef]

9. Liu, H.; Li, B.; Qiao, L.; Liu, J.; Ren, D.; Liu, W. miR-340-5p inhibits sheep adipocyte differentiation by targeting ATF7. Anim. Sci. J. 2020, 91, e13462. [CrossRef] [PubMed]

10. Dusaulcy, R.; Handgraaf, S.; Visentin, F.; Vesin, C.; Philippe, J.; Gosmain, Y. miR-132-3p is a positive regulator of alpha-cell mass and is downregulated in obese hyperglycemic mice. Mol. Metab. 2019, 22, 84-95. [CrossRef] 
11. Pan, Y.; Jing, J.; Qiao, L.; Liu, J.; An, L.; Li, B.; Ren, D.; Liu, W. MiRNA-seq reveals that miR-124-3p inhibits adipogenic differentiation of the stromal vascular fraction in sheep via targeting C/EBP $\alpha$. Domest. Anim. Endocrinol. 2018, 65, 17-23. [CrossRef]

12. Shi, T.; Yan, X.; Qiao, L.; Li, B.; Cheng, L.; Pan, Y.; Jing, J.; Cao, N.; Liu, W. MiR-330-5p negatively regulates ovine preadipocyte differentiation by targeting branched-chain aminotransferase 2. Anim. Sci. J. 2018, 89, 858-867. [CrossRef]

13. Deng, K.; Ren, C.; Fan, Y.; Liu, Z.; Zhang, G.; Zhang, Y.; You, P.; Wang, F. miR-27a is an important adipogenesis regulator associated with differential lipid accumulation between intramuscular and subcutaneous adipose tissues of sheep. Domest. Anim. Endocrinol 2020, 71, 106393. [CrossRef]

14. Kim, S.Y.; Kim, A.Y.; Lee, H.W.; Son, Y.H.; Lee, G.Y.; Lee, J.-W.; Lee, Y.S.; Kim, J.B. miR-27a is a negative regulator of adipocyte differentiation via suppressing PPARgamma expression. Biochem. Biophys. Res. Commun. 2010, 392, 323-328. [CrossRef] [PubMed]

15. Schlaepfer, I.R.; Joshi, M. CPT1A-mediated Fat Oxidation, Mechanisms, and Therapeutic Potential. Endocrinology 2020, 161, bqz046. [CrossRef]

16. Dang, Y.; Xu, J.; Zhu, M.; Zhou, W.; Zhang, L.; Ji, G. Gan-Jiang-Ling-Zhu decoction alleviates hepatic steatosis in rats by the miR-138-5p/CPT1B axis. Biomed. Pharmacother. 2020, 127, 110127. [CrossRef] [PubMed]

17. Deng, K.; Ren, C.; Liu, Z.; Gao, X.; Fan, Y.; Zhang, G.; Zhang, Y.; Ma, E.-S.; Wang, F.; You, P. Characterization of RUNX1T1, an Adipogenesis Regulator in Ovine Preadipocyte Differentiation. Int J. Mol. Sci. 2018, 19, 1300. [CrossRef]

18. Zhang, P.; Du, J.; Wang, L.; Niu, L.; Zhao, Y.; Tang, G.; Jiang, Y.; Shuai, S.; Bai, L.; Li, X.; et al. MicroRNA-143a-3p modulates preadipocyte proliferation and differentiation by targeting MAPK7. Biomed. Pharmacother. 2018, 108, 531-539. [CrossRef]

19. Cash, J.L.; Hart, R.; Russ, A.; Dixon, J.P.; Colledge, W.H.; Doran, J.; Hendrick, A.G.; Carlton, M.B.; Greaves, D.R. Synthetic chemerin-derived peptides suppress inflammation through ChemR23. J. Exp. Med. 2008, 205, 767-775. [CrossRef]

20. Li, X.; Wang, Y.; Guo, J.; Zhong, T.; Li, L.; Zhang, H.; Wang, L. Identification and expression patterns of adipokine genes during adipocyte differentiation in the Tibetan goat (Capra hircus). Gene 2018, 643, 17-25. [CrossRef] [PubMed]

21. Agarwal, V.; Bell, G.W.; Nam, J.-W.; Bartel, D.P. Predicting effective microRNA target sites in mammalian mRNAs. Elife 2015, 4, e05005. [CrossRef]

22. Narita, T.; Kobayashi, M.; Itakura, K.; Itagawa, R.; Kabaya, R.; Sudo, Y.; Okita, N.; Higami, Y. Differential response to caloric restriction of retroperitoneal, epididymal, and subcutaneous adipose tissue depots in rats. Exp. Gerontol. 2018, 104, 127-137. [CrossRef] [PubMed]

23. Richmond, S.R.; Carper, M.J.; Lei, X.; Zhang, S.; Yarasheski, K.E.; Ramanadham, S. HIV-protease inhibitors suppress skeletal muscle fatty acid oxidation by reducing CD36 and CPT1 fatty acid transporters. Biochim. Biophys. Acta 2010, 1801, 559-566. [CrossRef] [PubMed]

24. Patel, B.V.; Yao, F.; Howenstine, A.; Takenaka, R.; Hyatt, J.A.; Sears, K.E.; Shewchuk, B.M. Emergent coordination of the CHKB and $C P T 1 B$ genes in eutherian mammals: Implications for the origin of brown adipose tissue. J. Mol. Biol. 2020, 432, 6127-6145. [CrossRef] [PubMed]

25. He, W.; Gao, M.; Yang, R.; Zhao, Z.; Mi, J.; Sun, H.; Xiao, H.; Fang, X. The effect of CPT1B gene on lipid metabolism and its polymorphism analysis in Chinese Simmental cattle. Anim. Biotechnol. 2021, 1-13. [CrossRef] [PubMed]

26. Vantaku, V.; Dong, J.; Ambati, C.R.; Perera, D.; Donepudi, S.R.; Amara, C.S.; Putluri, V.; Ravi, S.S.; Robertson, M.J.; Piyarathna, D.W.B.; et al. Multi-omics integration analysis robustly predicts high-grade patient survival and identifies CPT1B effect on fatty acid metabolism in bladder cancer. Clin. Cancer Res. 2019, 25, 3689-3701. [CrossRef]

27. Van Weeghel, M.; Abdurrachim, D.; Nederlof, R.; Argmann, C.A.; Houtkooper, R.H.; Hagen, J.; Nabben, M.; Denis, S.; Ciapaite, J.; Kolwicz, S.C.; et al. Increased cardiac fatty acid oxidation in a mouse model with decreased malonyl-CoA sensitivity of CPT1B. Cardiovasc. Res. 2018, 114, 1324-1334. [CrossRef] [PubMed]

28. Hodson, L.; Rosqvist, F.; Parry, S.A. The influence of dietary fatty acids on liver fat content and metabolism. Proc. Nutr. Soc. 2020, 79, 30-41. [CrossRef]

29. Hess, B.W.; Moss, G.E.; Rule, D.C. A decade of developments in the area of fat supplementation research with beef cattle and sheep. J. Anim. Sci. 2008, 86, E188-E204. [CrossRef]

30. Chakrabarty, K.; Romans, J.R. Lipogenesis in the adipose cells of the bovine (Bos taurus) as related to their intramuscular fat content. Comp. Biochem. Physiol. B 1972, 41, 603-615. [CrossRef]

31. Justin, L.; Raymond, A.; John, M.; Masatoshi, M.; Lawrence, R.; Dale, R.; Jacqueline, W. Maternal and fetal growth, body composition, endocrinology, and metabolic status in undernourished adolescent sheep. Narnia 2007, 77, 343-350.

32. Sun, Y.; Wang, Q.; Zhang, Y.; Geng, M.; Wei, Y.; Liu, Y.; Liu, S.; Petersen, R.B.; Yue, J.; Huang, K.; et al. Multigenerational maternal obesity increases the incidence of HCC in offspring via miR-27a-3p. J. Hepatol. 2020, 73, 603-615. [CrossRef]

33. He, C.; Zhang, Q.; Sun, H.; Cai, R.; Pang, W. Role of miRNA and lncRNA in animal fat deposition-a review. Chin. J. Biotechnol. 2020, 36, 1504-1514.

34. Engin, A.B. MicroRNA and adipogenesis. Adv. Exp. Med. Biol. 2017, 960, 489-509. [PubMed]

35. Castaño, C.; Kalko, S.; Novials, A.; Párrizas, M. Obesity-associated exosomal miRNAs modulate glucose and lipid metabolism in mice. Proc. Natl. Acad. Sci. USA 2018, 115, 12158-12163. [CrossRef] [PubMed]

36. Chen, F.; Chen, Z.-Q.; Zhu, J.-J. Silencing of microRNA-27a facilitates autophagy and apoptosis of melanoma cells through the activation of the SYK-dependent mTOR signaling pathway. J. Cell Biochem. 2020, 121, 4694-4695. [CrossRef] 
37. Chen, H.; Zhang, Y.; Cao, X.; Mou, P. MiR-27a facilitates breast cancer progression via GSK-3ß. Technol. Cancer Res. Treat. 2020, 19, 1533033820965576. [CrossRef]

38. Choe, N.; Kwon, D.-H.; Ryu, J.; Shin, S.; Cho, H.J.; Joung, H.; Eom, G.H.; Ahn, Y.; Park, W.J.; Nam, K.-I.; et al. Targets ATF3 to reduce calcium deposition in vascular smooth muscle cells. Mol. Ther. Nucleic Acids 2020, 22, 627-639. [CrossRef]

39. Lin, X.-Z.; Luo, J.; Zhang, L.-P.; Wang, W.; Shi, H.-B.; Zhu, J.-J. MiR-27a suppresses triglyceride accumulation and affects gene mRNA expression associated with fat metabolism in dairy goat mammary gland epithelial cells. Gene 2013, 521, 15-23. [CrossRef]

40. Shi, C.; Huang, F.; Gu, X.; Zhang, M.; Wen, J.; Wang, X.; You, L.; Cui, X.; Ji, C.; Guo, X. Adipogenic miRNA and meta-signature miRNAs involved in human adipocyte differentiation and obesity. Oncotarget 2016, 7, 40830-40845. [CrossRef]

41. Yang, W.; Tang, K.; Wang, Y.; Zan, L. MiR-27a-5p increases steer fat deposition partly by targeting calcium-sensing receptor (CASR). Sci. Rep. 2018, 8, 3012. [CrossRef]

42. Lin, Q.; Gao, Z.; Alarcon, R.M.; Ye, J.; Yun, Z. A role of miR-27 in the regulation of adipogenesis. FEBS J. 2009, 276, 2348-2358. [CrossRef]

43. Teimouri, M.; Hosseini, H.; Shabani, M.; Koushki, M.; Noorbakhsh, F.; Meshkani, R. Inhibiting miR-27a and miR-142-5p attenuate nonalcoholic fatty liver disease by regulating Nrf2 signaling pathway. IUBMB Life 2020, 72, 361-372. [CrossRef]

44. Yu, Y.; Du, H.; Wei, S.; Feng, L.; Li, J.; Yao, F.; Zhang, M.; Hatch, G.M.; Chen, L. Adipocyte-derived exosomal MiR-27a induces insulin resistance in skeletal muscle through repression of PPAR $\gamma$. Theranostics 2018, 8, 2171-2188. [CrossRef]

45. Zou, B.; Ge, Z.; Zhu, W.; Xu, Z.; Li, C. Persimmon tannin represses 3T3-L1 preadipocyte differentiation via up-regulating expression of miR-27 and down-regulating expression of peroxisome proliferator-activated receptor- $\gamma$ in the early phase of adipogenesis. Eur. J. Nutr. 2015, 54, 1333-1343. [CrossRef]

46. Deng, K.; Ren, C.; Fan, Y.; Pang, J.; Zhang, G.; Zhang, Y.; You, P.; Wang, F. YAP1 regulates PPARG and RXR alpha expression to affect the proliferation and differentiation of ovine preadipocyte. J. Cell Biochem. 2019, 120, 19578-19589. [CrossRef] [PubMed]

47. Han, J.; Guo, T.; Yue, Y.; Lu, Z.; Liu, J.; Yuan, C.; Niu, C.; Yang, M.; Yang, B. Quantitative proteomic analysis identified differentially expressed proteins with tail/rump fat deposition in Chinese thin- and fat-tailed lambs. PLoS ONE 2021, 16, e0246279. [CrossRef] [PubMed]

48. García-Fernández, M.; Gutiérrez-Gil, B.; García-Gámez, E.; Sánchez, J.P.; Arranz, J.J. Detection of quantitative trait loci affecting the milk fatty acid profile on sheep chromosome 22: Role of the stearoyl-CoA desaturase gene in Spanish Churra sheep. J. Dairy Sci. 2010, 93, 348-357. [CrossRef]

49. Zhao, W.-S.; Hu, S.-L.; Yu, K.; Wang, H.; Wang, W.; Loor, J.; Luo, J. Lipoprotein lipase, tissue expression and effects on genes related to fatty acid synthesis in goat mammary epithelial cells. Int. J. Mol. Sci. 2014, 15, 22757-22771. [CrossRef] [PubMed] 